\section{What's Lurking Around the Corner? Polymyxin B Resistance Among Klebsiella pnemoniae carbapenemase-producing Enterobacteriaceae, and Others, as a Burden of Use}

To the Editor-The worldwide emergence of carbapenemresistant Enterobacteriaceae (CRE), a leading pathogen causing nosocomial infection, has challenged antimicrobial therapy. Many CRE isolates are only susceptible to polymyxins (polymyxin B and colistin [polymyxin E]), although resistance to this class of drug has drastically increased in recent years. ${ }^{1}$

Polymyxin resistance is a serious concern because this class of agent is considered the last-resort antimicrobial approach to multidrug-resistant gram-negative bacteria, including carbapenemase-producing Enterobacteriaceae (CPE), mostly Klebsiella pnemoniae carbapenemase (KPC) producers.

While attention has been focused on the widespread resistance to polymyxins among KPC producers, with worse associated outcomes, ${ }^{2}$ surveillance of polymyxin resistance rates among multidrug-resistant Enterobacteriaceae other than KPCproducers is lacking. Thus, because of the importance of polymyxin resistance today, we conducted a retrospective survey to assess the prevalence of polymyxin $\mathrm{B}(\mathrm{PMB})$ resistance among enterobacterial species recovered during a 1-year followup study.

The study included bacterial isolates consecutively collected from inpatients, from any clinical specimen, in a contemporary survey performed at a tertiary hospital in Southern Brazil, between January 1 and December 26, 2016. Identification of enterobacterial species as well as antimicrobial susceptibility testing was performed using an automated broth microdilution system (MicroScan; Beckman Coulter, Brea, CA). The minimum inhibitory concentration (MIC) of polymyxin B was confirmed using Etest (AB Biodisk, Solna, Sweden).

To attribute the resistance mechanisms for the enterobacterial species, a synergistic test was applied using phenylboronic acid and ethylenediaminetetraacetic acid to detect KPC and metallo- $\beta$-lactamase enzymes, respectively. Enzymatic inhibition testing with clavulanic acid and cloxacillin was used to detect extended-spectrum $\beta$-lactamases (ESBLs) and ampC enzymes, in that order, as previously described. ${ }^{3}$

In total, 1,746 enterobacterial species were enrolled during the study period. Overall, 1,166 isolates (66.8\%) were PMBsusceptible, and the remaining (33.2\%; 580 isolates) were PMBresistant isolates, regardless of the mechanism (ie, adaptive or intrinsic). Of the 580 PMB-resistant isolates, 404 (69.7\%) were species intrinsically resistant to this agent and, among them,
8 were carbapenem-susceptible ESBL-producing isolates (5 Morganella morgannii, 2 Serratia marcescens, and 1 Proteus mirabilis) and 4 isolates (all identified as $S$. marcescens) were also resistant to ertapenem and imipenem.

Among those 1,342 not intrinsically PMB-resistant isolates, $176(13.1 \%)$ showed an adaptive PMB resistance. Moreover, $44(25.4 \%)$ were KPC-producing isolates (the sole carbapenemase detected in this survey). Among them, $\mathrm{PMB}$ resistance was found in $165(37.2 \%)$ isolates; Klebsiella pneumoniae the only specie characterized as such. Of the enterobacterial isolates included in this study, PMB-resistant KPC-Kp represented $9.5 \%$ of all isolates. In addition, 1 carbapenem-resistant Enterobacter aerogenes proved to be PMB resistant (Figure 1).

Because the arsenal of new agents against multidrugresistant enterobacterial species has dried up, polymyxins have reemerged in clinical practice as agents of last resort, particularly for treatment of KPC infections in most Brazilian hospitals. ${ }^{4}$ By the way, little is known about the $\mathrm{PMB}$ resistance rates among organisms other than KPC ones, particularly those with any in vitro carbapenem susceptibility. Although less prevalent than KPC-Kp, these other PMB-resistant isolates were also detected in this study, and this fact may reflect a possible influence of PMB use in a setting with a high-level pressure for selecting resistant organisms, regardless of the mechanism (ie, adaptive or intrinsic) in the nosocomial environment.

Furthermore, this survey presents several important observations: First, KPC-Kp seems to be a carbapenemase-specie combination with fitness to develop PMB resistance attributed to prior use of this agent. ${ }^{3,5}$ Second, in this survey, 6 S. marcescens isolates (highlighting 4 of them with a carbapenem-resistance profile) were observed. Importantly, the intrinsic PMB resistance, especially that displayed by the $S$. marcescens specie, makes the acquisition of any carbapenemase encoding genes (particularly Serratia marcescens enzymes, SME) a worrisome event. This problem has already been emphasized in some countries, including Brazil. ${ }^{6,7}$ Third, PMB resistance does occur without carbapenem resistance, ${ }^{8}$ and this "silent resistance" is a concern because $\mathrm{PMB}$ is often used in clinical practice against those CRE. Fourth, although a small number of isolates was detected in this study, Enterobacter species are important in the acquisition of $\mathrm{PMB}$ resistance; they have been cited as a second epidemic agent emerging after the wave of a KPC-Kp. ${ }^{5}$

In conclusion, the increased occurrence of PMB-resistant microorganisms found along the PMB-susceptibility distribution is a worrisome trend. In this survey, the major concern was KPC-Kp, which presented higher PMB resistance rates. However, by becoming resistant to carbapenems (like ESBL-producing $S$. marcescens) or to polymyxins (like K. pneumoniae and Enterobacter spp), these threats deserve a careful surveillance to prevent their spread in healthcare settings. Strict protocols are urgently needed to guide polymyxin use. 


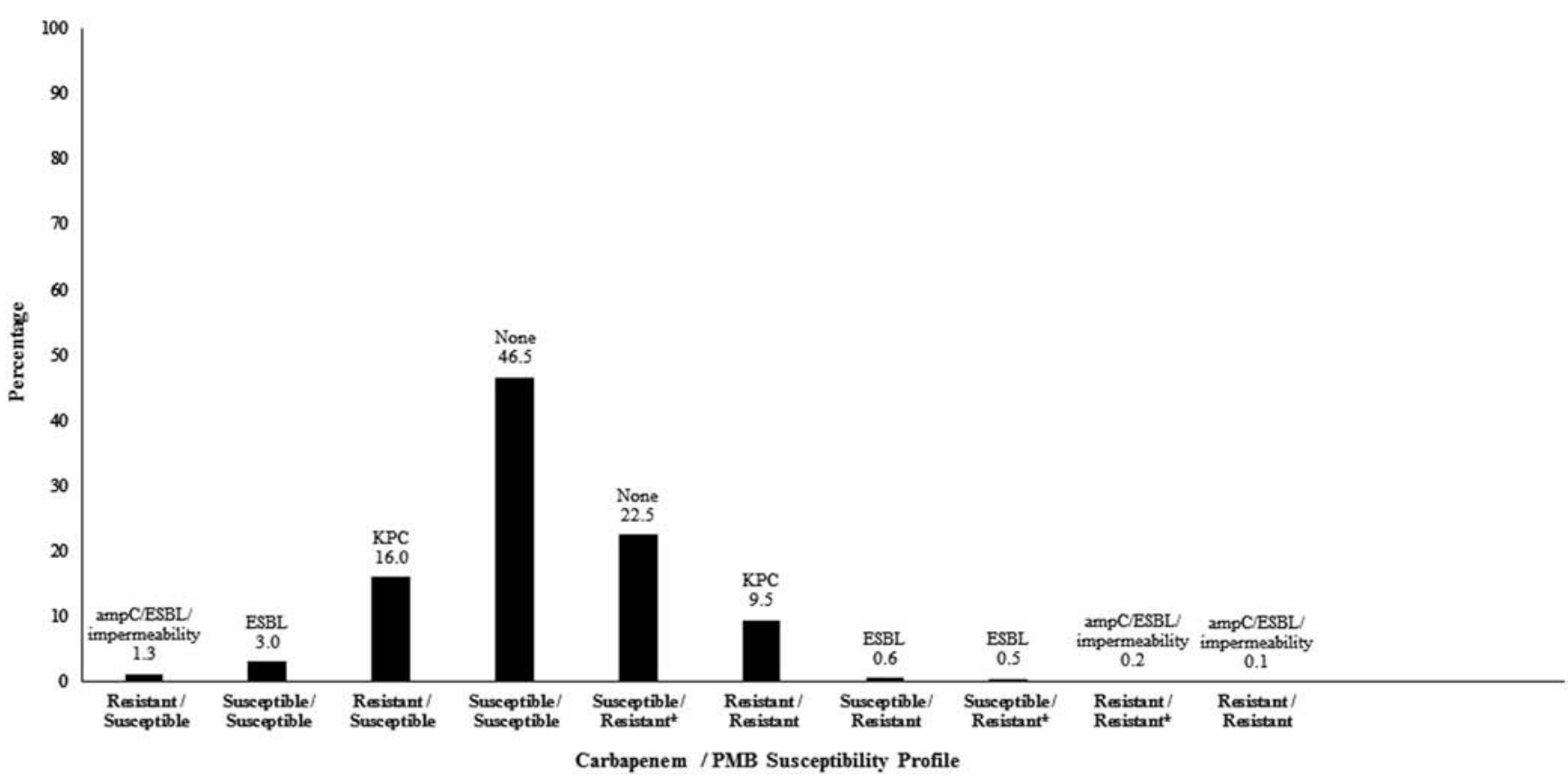

FIGURE 1. Crude prevalence of PMB susceptibility pattern of all enterobacterial species, distributed by the presence (or not) of any resistance mechanism to carbapenem and PMB agents, enrolled during the study period. Resistant with asterisk indicates intrinsic resistance to the antimicrobial agent.

\section{ACKNOWLEDGMENTS}

Financial support: No financial support was provided relevant to this article.

Potential conflicts of interest: The author reports no conflicts of interest relevant to this article.

\section{Leandro Reus Rodrigues Perez, $\mathrm{PhD}^{1,2}$}

Affiliations: 1. Hospital Mãe de Deus, Porto Alegre, Brazil; 2. Universidade Federal do Rio Grande do Sul, Porto Alegre, Brazil.

Address correspondence to Leandro Reus Rodrigues Perez, PhD, Microbiology Unit - Hospital Mãe de Deus, 286, José de Alencar Street, Porto Alegre, RS 90610-000 Brazil (leandro.reus@gmail.com).

Infect Control Hosp Epidemiol 2017;38:1120-1121

( 2017 by The Society for Healthcare Epidemiology of America. All rights reserved. 0899-823X/2017/3809-0021. DOI: 10.1017/ice.2017.135

\section{REFERENCES}

1. Jeannot K, Bolard A, Plésiat P. Resistance to polymyxins in Gram-negative organisms. Int J Antimicrob Agents 2017. doi: 10.1016/j.ijantimicag.2016.11.029.

2. Giacobbe DR, Del Bono V, Trecarichi EM, et al. Risk factors for bloodstream infections due to colistin-resistant KPC-producing Klebsiella pneumoniae: results from a multicenter case-controlcontrol study. Clin Microbiol Infect 2015;21:1106.e1-e8.

3. Rodrigues Perez LR. Carbapenem-resistant Enterobacteriaceae: a major prevalence difference due to the high performance of carbapenemase producers when compared to the nonproducers. Infect Control Hosp Epidemiol 2015;36:1480-1482.

4. Rodrigues Perez LR, Dias CG. Emergence of infections due to a polymyxin B-resistant KPC-2-producing Klebsiella pneumoniae in critically ill patients: What is the role of a previous colonization? Infect Control Hosp Epidemiol 2016; 37:240-241.

5. Perez LR. Does second place count? Lessons from a major discrepancy between carbapenem-resistant Klebsiella pneumoniae and carbapenem-resistant Enterobacter cloacae in a one-year follow-up study. Infect Control Hosp Epidemiol 2017. doi: 10.1017/ice.2017.36.

6. Hopkins KL, Findlay J, Meunier D, et al. Serratia marcescens producing SME carbapenemases: an emerging resistance problem in the UK? J Antimicrob Chemother 2017. doi: 10.1093/jac/dkw567.

7. Cayô R, Leme RC, Streling AP, et al. Serratia marcescens harboring SME-4 in Brazil: A silent threat. Diagn Microbiol Infect Dis 2017;87:357-358.

8. Perez LR. Is the polymyxin B resistance among multidrug-resistant Enterobacteriaceae (except for the carbapenemase-producing ones) a myth or a matter? Infect Control Hosp Epidemiol 2017;38:126-127.

To the Editor-The alarming increase in antibiotic resistance among enterobacterial species (ie, carbapenem-resistant Enterobacteriaceae [CRE]), mostly driven by the massive use of 